encouraging, and nurturing potential researchers and assessing the quality of their output. There is no better way to do this than by permitting a wide range of people to sample the world of research and test their product by submitting it to supervisors and peer reviewed journals.

Department of Epidemiology and Public Health, School of Health Care Sciences,

Medical School,

Medical School,

University of Newcastle upon Ty

1 Altman DG. The scandal of poor medical research. $B M \mathcal{F}$
1994;308:283-4. (29 January.)

2 Correspondence. The scandal of poor medical research. $B M f$ 1994;308:591-3. (26 February)

\section{Definition of the sudden infant death syndrome}

EDITOR,-The definition of the sudden infant death syndrome was called into question at the second international conference on the syndrome, in February 1992. As no consensus could be reached it was decided to keep the 1969 Beckwith definition: "the death of an infant or young child, which is unexpected by history and in whom a thorough necropsy examination fails to reveal an adequate cause of death."

The issue is what constitutes an "adequate cause of death." To base the definition of the sudden infant death syndrome on a diagnosis of exclusion is questionable. There is no current consensus on the minimum postmortem investigations that are needed to ensure that every cause of death has been excluded. There is also no consensus among pathologists on what is an adequate cause of death. The problem is not related to the identification of lesions, as every careful paediatric pathologist reports similar findings, ${ }^{1}$ usually noted as "insignificant lesions." This interpretation is the core of the problem and is not shared by French pathologists. They believe that the pathological findings can be considered to be an adequate cause of death in three quarters of the cases diagnosed as the sudden infant death syndrome in many countries. ${ }^{23}$

The main difference between the French and other approaches to sudden infant death is the systematic protocol that involves many specialists at the time of death. ${ }^{4}$ Any infant who dies suddenly is brought to the emergency room and dealt with in the same way as an acutely ill child would be. Biological and microbiological samples are collected within 10 hours of the last sighting of the infant alive in $90 \%$ of cases. Paediatricians direct the sampling process and collect the paediatric and obstetric data, and microbiologists play a part well before the necropsy protocol starts. The extensive necropsy protocol is always performed by a paediatric pathologist with a major interest in the sudden infant death syndrome. ${ }^{4}$ All findings are reviewed at a multidisciplinary conference, and the decision concerning the cause of death is made there. This approach is very different from many investigations into sudden infant death that have been reported in the United States, for example.'

The definition of the sudden infant death syndrome is crucial as many epidemiological data will be derived from infants labelled as having died of the syndrome by a bureaucratic, sometimes ill equipped system. These so called epidemiological data may misdirect researchers toward irrelevant protocols. It has been reassuring for parents to be given something that they consider to be a diagnosis (that is, the sudden infant death syndrome), and such a positive impact must be taken into consideration. Therefore, we suggest a modification of the definition of the syndrome. The sudden infant death syndrome should be diagnosed in every case of cot death fulfilling the classical clinical criteria for the syndrome (a 1 month to 1 year old infant, clinically well before the death or with only minor illness, is put to sleep and found dead), with a subsequent classification according to the main postmortem findings (for example, the sudden infant death syndrome with myocarditis). This systematic indication in every report of the main two or three findings, as a minimum requirement, may give more substance and medical usefulness to the term sudden infant death syndrome.

CAROLINE RAMBAUD Associate professor

Anatomie-Pathologique,

Hôpital Necker-Enfants Malades,

75743 Paris Cedex 15,

France

CHRISTIAN GUILLEMINAULT Professor

Sleep Disorder Clinic

Palo Alto,

CA 94304, USA

PETER E CAMPBELI

ictoria Institute of Forensic Pathology, Physician

South Melbourne,

Victoria 3205,

Australia

1 Valdés-Dapena $M$, McFeeley PA, Hoffman $\mathrm{HJ}$, Damus $\mathrm{KH}$ Franciosi RR, Allison DJ, et al. Histopathology atlas for the sudden infant death syndrome. Washington, DC: Armed Force Institute of Pathology, American Registry of Pathology, and National Institute of Child Health and Human Developmen 1993.

2 Rambaud C, Cieuta C, Canioni D, Rouzioux C, Lavaud J, Hubert $\mathrm{P}$, et al. Cot death and myocarditis. Cardiology in the Young 1992;2:266-71.

Chéron G, Rambaud C, Rey C, Mahut B, Canioni D, Lavaud J, et al. Morts subites au berceau. Expérience d'un centre de référence, 1986-1 1991. Arch Fr Pediatr 1993;50:293-9.

Rambaud C, Imbert MC. Protocole d'autopsie d'une mort subite du nourrisson Ann Pathol 1993;2:131-4.

5 Nowak R. Investigating infant deaths: an ill-equipped system struggles to cope. Fournal of National Institute of Health struggles to cope. 7 Research 1993;5:29-31.

\section{Doctors and homosexuality}

\section{Homosexuals deserve better from doctors}

EDITOR,-The correspondence ${ }^{1}$ concerning Lynn Rose's paper on homophobia ${ }^{2}$ was a major topic of the discussion at a recent monthly meeting of the Lesbian and Gay Health Workers Association. Members of the association represent a wide range of health workers, and we believe that we can offer a consolidated opinion for all homosexuals working in health care.

We believe that it is essential to identify the key facts in the debate. Homophobia may be the wrong word for the attitude of malice towards homosexual people, but nothing can disguise the fact such an attitude can be found throughout the health service in a scattering of staff. Fortunately, an equal number of heterosexuals will support and defend the right of equality. Homosexuals who are struggling to cope with their sexuality perceive a bigger problem than really exists, but doctors are driven to contemplate suicide by trying to resolve their feelings in what they believe is a hostile profession. We have never heard of homophobic doctors considering the same.

The criticism of Rose's methods is valid, but homophobia is extremely difficult to prove or quantify. Homophobia is indicated by attitudes more commonly than it is by actual actions. Isolating a patient in a side room because "the other patients wouldn't want a shirt-lifter on the ward" is homophobic, but allowing a patient to have a side room so that he and his partner can show affection like every other couple is compassionate.

Homophobes who claim that homosexuality will affect someone's ability to work in the health service imply that they consider their heterosexua attractions to be essential to correct professional behaviour, which is ludicrous. But if sexuality is irrelevant to the practice of medicine the reason for our association might be questioned. Sexuality should not be the overriding component of anyone's life, but it does affect our social life. Health staff talk socially about their partners. We would like to be able to do the same without fear of affecting our job prospects. Being gay is not like smoking, drinking, or riding motorbikes, for those are acts of choice; unlike colour or gender, being gay is not immediately identifiable to others. It often takes time for us to identify what we are. It is always difficult, and sometimes almost impossible, to explain, but a life of secrecy is an intolerable strain. We accept that some people cannot approve, but we resent the active discrimination in parts of the profession and call on those who abhor such attitudes to challenge them whenever they occur. Gays and lesbians deserve better from their doctors, and those of us who have not yet come out are sick of living a lie.

VICTOR CALIAND Secretary TIM WORDEN Founding membe BILL WILLIAMS Treasurer M C HAYES-ALLEN

Lesbian and Gay Health Workers Association,

PO Box 153,

Manchester M60 1LP

1 Correspondence. Doctors and homosexuality. $B M F$ 1994;308: 854-5. (26 March.)

2 Rose L. Homophobia among doctors. BMf 1994;308:586-7. (26 February.)

\section{Doctors could make life easier}

EDIToR,-I wish to respond to the correspondence on my paper on homophobia among doctors.' The paper is an extrapolation from a paper for a masters degree of 21000 words entitled "Doctors divide? Doctors and sexuality in the era of HIV and AIDS." A copy of this is at South Bank University's library.

The questions regarding sample size and selection, subjectivity and objectivity, lifestyle effects on medical treatment, the reasons for my involvement in the type of research, ethics, and so on are all clarified in the original document, as is a definition of homophobia, which seems to be a fairly new word in English dictionaries. The statement "blatantly homophobic" was a direct quotation from one of the respondents.

The article was an attempt to show that the medical profession could make life easier for their colleagues, themselves, and patients with HIV infection and AIDS. Further research is certainly needed, and is also requested in the document.

London NW3 5PB

LYNN ROSE

1 Correspondence. Doctors and homosexuality. BMF 1994;308: 854-5. (26 March.)

\section{Depression and impotence}

EDrTOR,-For a long period erectile dysfunction (impotence) was assumed to have primarily a psychological basis. Except in cases of severe neurological impairment and endocrinopathies, the management of the disorder was handed to psychotherapists, marriage counsellors, or whoever else might be found to offer guidance. In the 1960s Masters and Johnson set out a view of the nature of sexually dysfunctional disorders together with a plan of treatment that was persuasive and partially successful'; they provided a model for clinics treating sexual dysfunction, which increased in numbers. Yet Masters and Johnson admitted that, of all the sexual dysfunctions, erectile dysfunction was the most resistant to 